\title{
Neutropenia autoinmune infantil
}

\author{
Ernesto Ríos L.'; Sonia Arredondo V. ${ }^{2}$
}

\section{Autoimmune neutropenia}

\begin{abstract}
Results of a prospective follow up of 15 children with chronic neutropenia lless thon 1000 neutrophils/ul of peripheral b'ood, thict lasted more than 15 dayst are described. At diagnosis all patients had absolute blood neutrophil counts less han $1000 / \mu \mathrm{ll}$. The following lest were performed to all of them: repealed blood leukocyte and differencial counts during the period of neutropenia, bone matrow aspirate examination and serum anti-neutrophil antibodies by indirect immunoflurescent rest. Mean age at diagnosis was 8 month, range 5 to 18 monlhs. All patients had positive serum anlineutrophil anlbodies, of litles that ranged from $1 / 20$ to $1 / 80$, mean $1 / 55$. Absolute neutrophil blood cell counts recovered spontaneously in all potienls 4 to 63 months, median 15 monlhs, after detection of neutropenia. Several bocierial infectious episodes of differenl frequency, ethiolagy and severity were recorded in mine patients, bul all resolved favourably with antigiotic rearment. Six palients had no infections al all along the whole neutropenic period. Autoimmune neutropenia is probably the mosl frequent couse of infaril's chronic neutropenia. It usually recovers sponloneously in variable lengths of time, and requires conservative manogement.
\end{abstract}

[Koy words: neutropenia, autoimmunity, auloanlibodies, autoimmune diseases.)

La neutropenia se define como un número absoluto de neutrofilos menor a $1500 / \mu$ en el hemograma. La neutropenia no es infrecuente en pediatría, pues suele acurrir en la etapa inicial de las infecciones virales, como también en los primeros días de evolución de infecciones bacterianas severas, que pueden agotar la reserva medular, por el gran requerimiento de neutrófilos en los tejidos y el tiempo de latencia que tiene la médula granulopoyética para aumentar eficazmente su producción'.

Sin embargo la neutropenia de más de 15 días de duración es poco usual y en este caso deben descartarse problemas como mieloptisis, mielodisplasia, reacciones a drogas, afecciones de mecanismo inmunitario, defectos congénitos. El estudio de la neutropenia persistente puede ser muy complejo, en él ocupa lugares predominantes el análisis del mielograma. La historia clínica, el tiempo transcurrido desde el inicio de la enfermedad, la ingestión de medicamentos,

1. Departamento de Pediatrfa. Hospital Luis Calvo Mac. kenna. Facultad de Medicina, Campus Oriente. Universidad de Chile.

2. Tecnologa Médico. Departamento de Pediatría. Hospital Luis Calvo Mackenna. Facultad de Medicina. Carnpus Oriente. Universidad de Chile. orientan hacia diferentes posibilidades etiopatogénicas que requerirán estudios específicos para cada situación.

En las neutropenias crónicas suelen estar involucrados mecanismos inmunitarios. En efecto, en algunas de las causadas por drogas, estas sustancias actúan produciendo haptenos o complejos inmunes; en recién nacidos, la sensibilización materna y traspaso de anticuerpos puede producir neutropenia aloinmune; finalmente, enfermedades debidas a autoinmunidad son capaces de producir neutropenia, que puede ser secundaria a la enfermedad basal -como el lupus diseminado o el síndrome de Felty-o primaria, afectando, en este caso exclusivamente a los neutrófilos ${ }^{2-6}$.

El proposito de esta publicación es describir los procedimientos para el diagnóstico, el tratamiento y la evolución en lactantes con neutropenia crónica, en que el mecanismo etiopatogérico es de autoinmunidad primaria.

\section{Material y Métodos}

Se estudiaron prospectivamente quince lactantes, para los cuales los criterios iniciales de inclusión fueron la presencia de neutropenia con número absoluto de neutrofilos 
(RAN) menor a 1000 jul de sangre periférica, de más de 15 días de duración. En todos se hizo una historia detallada de episodios infecciosos, medicamentos recibidos y vacunaciones en el mes que precedjo al inicio de la enfermedad. Ellos fueron examinados, estudiados y controlados por las mismas personas. A todos se les efectuaron recuentos de leucocitos y fórmula reiteradamente durante el curso de la enfermedad, mielograma y búsqueda en el sueto de anticuerpos antineutrofilos. Su evolución fue seguida con conteos mensuales de la serie blanca durante el primer año de control y uno cada dos o tres meses posteriormente. Además se efectuaron todos jos exámenes necesarios para identificar la causa de los episodios febriles que ocuriesen. Se consideró que un paciente se había recuperado de la neutropenia cuando se registraron al menos tres exámenes de serie blanca contiguos con RAN normal, situación en la cual este recuento se repitió mensualmente.

La determinación de anticuerpos antineutrófilos en suero se hizo por el método de inmunofluorescencia indirecta. en el cual se áslan neutrófilos de una persona normal, en Ficoll-Hypaque con doble gradiente de densidad ${ }^{7,8}$ : luego se Javan las células en un tampón con paracetaldehído, con el fin de eliminar las inmunoglobulinas inespecficas de la membrana. A continuación se incuban los neutrófilos con el suero en estudjo, se lavan y finalmente se incuban con el conjugado antigamaglobulina humana (IgG, $\operatorname{lgA}, \operatorname{IgM}$ ) obtenido en cabras y marcado con isoniocianato de fluoresceína (FITC) (Kallestead Laboratories). La lectura se efectúa en microscopio para intnunofluorescencia. El suero de cada paciente se diluye progresivamente para titular la concentración de anticuerpos, considerándose la última dilución positiva para expresar el título señalado en los resultados. Las muestras de suero para determinar estos anticuerpos se guardaron congeladas hasta reunir varias de ellas antes de procesarlas, dados la complejidad de la técnica, el coste de los reactivos y la conveniencia de obtener el mejor aprovechamiento posible de ellos.

En algunos pacientes, como parte del estudio de una neutropenja crónica y para descartar posibles neutropenias congénitas, se efectuaron, además, conteos absolutos seriados de neutrófilos dos veces por semana durante cuatro a ocho semanas, en busca de neutropenita cíclica"; desmarginación de neutrófilos en la sangre mediante prueba de adrenalina ${ }^{\text {'b }}$, para despistar psewdoneutropeniz; inmunoglobulinas séricas, para eliminar neutropenia con inmunodeficiencia (enfermedad de Brulon) ${ }^{l 1}$; búsqueda de grasas en deposiciones, para descartar la neutropenia con compromiso de páncreas exocrino (sindrome de Shwachman) ${ }^{12}$ y recuento de neutrofilos a los padres y hermanos, para excluir la neutropenia familiar ${ }^{13}$.

Durante la evolución, los pacienles fueron mantenidos en aislamiento relativa en su casa, evitándoles contactos con orros nifos enfermos, asistencia a lugares públicos con gente y ofreciéndoles comjdas cuyos ingredientes fueran cocidos. Los episodios infecciosos fueron tratados de acuerdo a su etiologia siguiendo criterios pediátricos generales y no se les efecturon tratamientos espectificos destinados a controlar la enfermedad.

\section{Resultados}

Los quince pacientes eran lactantes, Ia mediana de su edad era 8 meses, márgenes 5 a 18 meses, nueve eran mujeres. Doce habían tenido síntomas que hacían sospechar infecciones en el mes previo a la constatación de la neutropenia en el hemograma, sugerentes de ser episodios virales, destacando entre ellos los de la vía aérea, como resfrío común, gripe, bronquitis obstructiva, gingivoestomatitis y neumonitis $y$, en dos casos, exantema súbito. En cinco se registraban antecedentes de diarrea independiente o concomitante con otras manifestacjones de infección (en uno de ellos se identificó rotavirus, en otro Escherichia coli enteropatógena). En sólo dos lactantes no se registraron síntomas, pero ambos habían recibido vacunas con virus vivos (uno antisarampión, otro la combinación contra sarampión, parotiditis y rubéola) y en el segundo se agregó diarrea después de la inmunizacion. Antes de comprobarse la neutropenia y en relación con los episodios presuntamente infecciosos mencionados, nueve pacientes recibieron medicamentos que potencialmente pueden producir daño hematológico, alterando la granulopoyesis ${ }^{14}$ : dipirona en seis casos, naproxeno, cotrimoxazol, ibuprofeno o cloranfenicol, un paciente cada fármaco (uno de los pacientes recibió dos medicamentos potencialmente causantes).

Por lo general el examen físico de los lactantes era normal, excepto en aquellos que estuviesen cursando con un episodio febril y algún signo especffico. Ningún paciente tuvo esplenomegalia al ingreso o durante la evolución.

Los resultados de los hemogramas y anticuerpos antineutrófilos séricos se describen en la tabla, haciéndose notar que el número de leucocitos estaba entre los márgenes normales, que el número absoluto de neutrófilos era menor a 500 células/ $\mu l$ de sangre, y por lo tanto existía neutropenia severa, mientras el recuento absoluto de monocitos estaba dentro de los límites de lo normal. El mielograma mostró en doce de los quince pacientes, hiperplasia de la serie granulocitica y una secuencia madurativa alterada, en la que predominaban promielocitos y mielocitos mientras el número de juveniles, baciliformes y segmentados era progresivamente menor, siendo -estos últimos- escasos, si bien presentes. En los otros tres lactantes afectados había, en cambio, hipoplasia mieloide leve con idéntica alteración de la secuencia madurativa descrita en los demás niños. El aspecto señalado del mielograma no es específico para el diag- 


\section{Tabla}

Resultados iniciales de laboratorio

\begin{tabular}{lccc}
\hline & $\overline{\mathbf{x}}$ & Margenes \\
\hline Recuento de leucocitos (x $\left.10^{3} / \mu\right)$ & 8,00 & $4,10-12.5$ \\
Recuento absoluto de neutófilos $(x$ & $\left.10^{3} / \mu \mathrm{l}\right)$ & 0,49 & $0,07-0,91$ \\
Recuento absoluto de monocitos (x $\left.10^{3} / \mu 1\right)$ & 0,70 & $0,18-1,63$ \\
Anticuerpos antineutrofilos (tílulo) & $1 / 55$ & $1 / 20-1 / 80$ \\
\hline
\end{tabular}

nóstico, porque se encuentra tanto en neutropenias producidas por drogas como por infecciones bacterianas severas, además de cualquier causa inmunitaria, no sólo la autoinmune, pero permite descartar las neutropenias tumorales y algunas congénitas. El promedio de los títulos de anticuerpos antineutrófilos de 1/55 no es muy elevado.

En nueve pacientes se efectuaron los estudios adicionales para descartar la presencia de una neutropenia congénita (muchos de estos exámenes se realizaron mientras se esperaban las determinaciones de las concentraciones séricas de anticuerpos antineutrófilos), todos con resultados normales.

La duración de la neutropenia varió ampliamente de un caso a otro, con mediana de 15 meses y márgenes de 4 a 63 meses, pero finalmente todos los pacientes se recuperaron espontáneamente, ya que no se intentó ningún tratamiento específico para ella. No se encontró relación entre dicha duración y el título de anticuerpos antineutrófilos. Seis pacientes permanecieron asintomáticos durante el curso de la neutropenia. Los otros nueve sufrieron episodios de infecciones, que en seis fueron repetidos y en tres únicos. Las infecciones estuvieron constituidas por otitis, laringitis, faringitis, bronquitis, gingivoestomatitis e infecciones cutáneas, todas fueron tratadas con antibióticos en combinación, de acuerdo a las normas pediátricas para pacientes neutropénicos, y todos ellos se restablecieron adecuadamente, sin que alcanzaran mayor gravedad. La forma más frecuente fue otitis. En tres pacientes se usó cotrimoxazol (20 mg - $\mathrm{kg}$ - día de trimetoprim, dividido cada 12 horas por vía oral), para prevenir infecciones, con resultados no evaluables, ya que dos de estos casos sufrieron repetidas infecciones, mientras el otro se mantuvo libre de éstas.

\section{Comentario}

En e] momento de su detección, la etiología de una neutropenia es generalmente confusa. Muchas veces se trata de un paciente febril con antecedente de haber recibido medicamentos potencialmente $t 6 x i c o s$ para la médula ósea, en cuyo hemograma se constata la neutropenia. Para orientarse en la etiopatogenia del problema el mielograma es fundamental. La historia detallada es muy importante para determinar la evolución previa y son de gran ayuda los hemogramas anteriores, antecedentes de episodios febriles repetidos, los medicamentos tomados, su dosis y el número de veces, la exposición a tóxicos volátiles, las vacunas recibidas. En la experiencia que se comenta todos estos hechos se detectaron en varios pacientes.

Una situación que tiende a producir confusiones son las variaciones del RAN en los controles sucesivos de hemogramas de estos enfermos, que muchas veces hacen plantear la posibilidad de neutropenia cíclica, como ocurrió con seis de los lactantes de este estudio. La unica manera de confirmar o descartar este inusual trasłorno congénito es haciendo recuentos en serie del RAN y trazar la curva correspondiente, lo que permite observar si existe o no la variación cíclica y ordenada de los neutrófilos que lo caracteriza. En la neutropenia autoinmune, debido a la relativamente baja concentración de anticuerpos, se producen aumentos de los neutrófilos ante estímulos infecciosos, lo que confunde ambas situaciones. También se observan oscilaciones del RAN hacia el final de la neutropenia autoinmune, produciendose luego la estabilización definitiva de los conteos entre los márgenes normales, criterio con el cual se define el término de la enfermedad. Durante la evolución es frecuente observar la presencia de eosinófilos.

Los resultados normales de la prueba de adrenalina para la demarginación de los neutrófilos, en los tres pacientes en que se hizo, no concuerdan con lo publicado por otros autores ${ }^{6}$, sin embargo tampoco parece clara la razón por la que debiesen alterarse las cantidades de neutrófilos circulantes y marginados (normalmente $50 \%$ cada uno) a pesar de la neutropenia autoinmune. En la prueba de demarginación normal el recuento de neutrofilos circulantes aumenta al doble, como ocurrió en estos tres pacientes. En 
todo caso, el pequeño número de casos en que se efectú la prueba impide sacar conclusiones definitivas; además uno de ellos tenía, circunstancialmente, un RAN normal al hacer la prueba.

La prednisona en el tratamiento de la neutropenia autoinmune ha dado resultados poco alentadores o solamente parciales ${ }^{15}$, a lo que se agrega el aumento del riesgo de infecciones severas que implica el uso de corticoesteroides ${ }^{6}$. Con gamaglobulina endovenosa en dosis de $0,5 \mathrm{~g} \cdot \mathrm{kg}$ de peso $\cdot \mathrm{dia}$, se ha conseguido elevaciones transitorias de los neutrófilos por 7 a 10 días $y$, ocasionalmente, revertir la situacion ${ }^{16,17}$. En el presente estudio se observó la evolución espontánea, sin que ocurrieran episodios infecciosos severos. Existe consenso que en caso de una enfermedad grave se debe recurrir a gammaglobulina endovenosa. También ha sido usado el cotrimoxazol como tratamiento preventivo de infecciones mientras se mantiene la neutrope$\mathrm{nia}^{18}$, como se hizo en este estudio con tres pacientes, no siendo orientadores sus resultados.

Los neutrofilos tienen antígenos específicos designados como NA1 - NA2, NB1 - NB2, $\mathrm{NCl}$, ND1 y NEI en los cinco locus descritos hasta ahora ${ }^{18}$. En la neutropenia inmune, la mayoría de los autoanticuerpos están dirigidos contra los antígenos NA1 y NA2 ${ }^{19}$. En los pacientes aquí descritos no fue posible realizar el estudio correspondiente, sin embargo, los anticuerpos contra el antígeno NB2 son aglutininas y no son detectados por la técnica de inmunofluorescencia indirecta, por lo tanto en estos pacientes, al menos, se puede concluir que no hubo anti NB2.

Se han descrito antígenos específicos en células inmaduras de la serie granulocítica ${ }^{20}$, esto podría explicar que en algunos pacientes se observe hipoplasia de la serie granulocítica de la médula ósea, al actuar el anticuerpo en células troncales, además de mantenerse la alteración de la secuencia madurativa, como ocurrí en tres de los pacientes aquí presentados, que mostraron esta imagen en el mielograma.

Para explicar la relativa frecuencia de la neutropenia autoinmune en lactantes se ha formulado como hipotesis que se conjugarian una inmadurez -descrita en los linfocitos T supresores- con una respuesta anómala, a un factor externo desencadenante, a través de la infección viral o drogas, sobre los linfocitos $\mathrm{T}$ y un factor genético predisponente, ya que se ha demostrado la asociación de neutropenia autoinmune debida a antígenos NAl y HLA-DR2 ${ }^{1 B}$. Por orra parte, se ha especulado que coincidiendo con la culminación del desarrollo de la función supresora de los linfocitos $\mathrm{T}$, alrededor de los tres años de edad, se produciría la recuperación espontánea de la neutropenia autoinmune 21 .

\section{Resumen}

Se describen quince lactantes que sufrieron neutropenia crónica, con recuentos absolutos de neutrófilos menores a $1000 / \mu l$ de sangre al ingreso. En todos los pacientes se efectuaron recuentos de leucocitos y fórmula leucocitaria repetidamente durante la enfermedad, estudio de médula ósea y mediciones de anticuerpos antineutrofilos por inmunofluorescencia indirecta. La mediana de la edad al diagnóstico era $8 \mathrm{me}$ ses, márgenes 5 a 18 meses. La enfermedad fue comprobada en todos los pacientes por anticuerpos antineutrófilos circulantes en el suero a títulos entre $1 / 20$ y 1/80. Todos los casos se recuperaron espontáneamente, sin mediar tratamientos "específicos" para la neutropenia, al cabo de 4 a 63 meses, mediana de 15 meses, desde la detección del trastorno. Seis pacientes sufrieron episodios reiterados de infección durante el curso de la neutropenia, tres uno solo. Todos los casos respondieron a los tratamientos convencionales con atibióticos. Seis pacientes fueron asintomáticos. La neutropenia autoinmune es probablemente la causa mas frecuente de neutropenia crónica del lactante. Evoluciona en plazos variables a la mejoría espontanea, requiriendo de tratamiento conservador.

(Palabras clave: neutropenia, autoanticuerpos, autoinmunidad, enfermedades autoinmunitarias.)

\section{Referencias}

1. Ríos E: Control y alteraciones de la granulopoyesis: neutropenia - neutrofilia. Rev Chil Pediatr 1986; 57: 79-85.

2. Salama A and Mueller-Eckhardt $C$ : Inmune-mediated blood cell dyscrasias related to drugs. Semin Hematol $1992 ; 29: 54-63$.

3. Boxer LA, Yokoyama $M$ and Lalezari P: Lsoimmune neonatal neutropenia. 3 Pediatr 1972; 80: 783-787. 
4. Ros $E$. Heresi $G$ and Arévalo $M$ : Familial alloimmune neutropenia of NA2 specificity. Am I Pediatr Hematol Oncol 1991; 13: 296-299.

5. Logue GL and Shimm DS: Autoimmune granulocy. topenia. Annu Rey Med 1980; 31: 191-200.

6. Lalezari $P$, Manoochehr $K$ and Petrosova $M$ : Autoimmune neutropenia of infancy. J Pediatr 1986; 109: 764-769.

7. Verheugt $F W$, von dem Borne AE, var Noord-Bok horst, et al: Autoimnune granulocytopenia: the detection of granulocyte autoantibodies with the immunofluorescence test. Br J Haematol 1978; 39: 339-350.

8. Madyastho $P$. Madyostha $X R$. Wade $T$, et al: An improved method for rapid layering of Ficoll-Hypaque double density gradients suitable for granulocyte separation. J Immunol Methods 1982; 48: 281-286.

9. Dale DC, and Hammand WP: Cyclic neutropenia: a clinical review. Blood Revicws 1988; 2: 178-185.

10. Joyce $R$. Buggs DR. Hasiva V, et al.: Marginal neutrophil pool size in normal subjets and neutropenic patients as measured by epinephrine infusion. J Lab Clin Med 1976; 88: 614-626

11. Rosen FS. Cooper MD and Wedwood RJ: Medical progress: The primary immunodeficiencies. N Engl J Med 1984: 31 1: 235-239.

12. Agett PJ, Cavanagh NP, Mathew DJ, et al.: Shwachman syndrome. Arch Dis Child 1980; 55: 331-347.
13. Bjure $J$, Nilsson $L R$ and Plum CM: Familial neutropenia possibly caused by deficiency of a plasma factor. Acta Paediatr Scand 1962; 51: 497-501.

14. Young $G A$ and Vincent $P C_{\text {: }}$ Drug induced agra+ nulocytosis. Clin Haematol 1980; 9:483-504,

15. Lalezari $P$. Jiong $A F$, Yegen $L$ et al.: Chronic autoimmune neutropenia due to anti-NA2 antibody. N Engl J Med 1975; 293; 744-747,

16. Hilgartner $M W$ and Bussel J: Use of intravenous gamma globulin for the treatment of autoimmune neutropenia of childhood and autoimmune hemolytic anemia. Am J Med 1987; 83 (suppl 4A): 25-29.

17. Bussel J, Lalezari P, Hilgoriner $M W$ et al.: Reversal of neutropenia with intravenous gamma globulin in autoimmune neutropenia of infancy. Blood 1983; 62: $398-400$.

18. Bux $J$, and Mueller-Eckhardt $C$ : Autoimmune neutropenia. Semin Hematol 1992; 29: 45-53.

19. Madyastha PR, Kyong CU. Darby $C P$ et al.: Role of neutrophil antigen NAl in an infant with autoimmune neutropenia. Am J Dis Child 1982; 136: 718721.

20. Valbonesi $M$, Campbell A+ Marazzi $M G$ et al.: Chronic autoimmune neutropenia due to anti-NAL antibody. Vox Sang 1979: 36: 9-12.

21. Shastri $K A$ and Logue GL: Autoimmune neutropenia. Blood 1993: 81: 1984-1995.

\section{AVISO A LOS AUTORES}

Por acuerdo del Comité Editorial, la Revista Chilena de Pediatría devolverá sin tramitar todos los trabajos que no den estricto cumplimiento al Reglamento de Publicaciones y a las Instrucciones de los Autores que se editan en cada número de la Revista. 